\section{Marion ERNWEIN}

Département de géographie et environnement Université de Genève

\section{Joëlle \\ SALOMON CAVIN}

Institut de géographie et durabilité Université de Lausanne Géopolis

Mots-clés : ville, agriculture, aménagement, urbanisme, agriculture urbaine, agrarisation

Keywords: city, agriculture, spatial planning, urban planning, urban agriculture, agriculturalisation

\section{Au-delà de l'agrarisation de la ville : l'agriculture peut-elle être un outil d'aménagement urbain ? Discussion à partir de l'exemple genevois}

Résumé

Deux processus permettent de décrire la relation contemporaine entre agriculture et ville : d'une part l'urbanisation de l'agriculture et d'autre part l'agrarisation de la ville. Le premier terme de la proposition parait relativement évident et renvoie notamment aux effets de l'étalement urbain sur les espaces agricoles, mais le second peut paraitre plus surprenant et mérite que l'on s'y arrête. Ainsi, après avoir identifié les caractéristiques de l'agrarisation émergente de la ville, nous nous intéressons aux formes qu'elle prend dans le contexte genevois. Enfin, nous mettons en évidence les obstacles et limites qui constituent aujourd'hui un frein au processus d'agrarisation de la ville. Ce faisant, nous interrogeons le rôle de l'agriculture comme outil d'aménagement.

\section{Abstract}

The contemporary relationship between city and agriculture is undergoing two processes: on the one hand the urbanisation of agriculture and on the other hand the agriculturalisation of the city. The first proposition sounds obvious and refers to the effects of urban sprawl. The agriculturalisation of the city may be more surprising a proposition, and is worth explaining. After identifying the main characteristics of the agriculturalisation of the city we propose an analysis of the process of agriculturalisation of Geneva, Switzerland. In a last part, we put forth the limitations to the process, and by so doing, critically examine the role of agriculture as a tool for urban planning.
1 - Un groupe de travail réunit des représentants de différents services de I'adminsitration cantonale autour de la question générale de l'agriculture urbaine; un second groupe de travail réunit des représentants de différents services municipaux autour de la place à donner au jardinage urbain dans les politiques.
La question de I'institutionnalisation de l'agriculture urbaine et de sa transformation en outil de I'action publique que pose ce numéro paraît trouver un écho croissant auprès des collectivités. En témoigne, par exemple, le colloque "Villes jardinées et initiatives citoyennes ", qui a réuni à Strasbourg en octobre 2012 plusieurs centaines de représentants de communes françaises et de personnes issues du milieu du jardinage associatif et de l'agriculture urbaine, dans le but de s'interroger collectivement sur les passages entre les pratiques associatives et professionnelles.

Genève ne fait pas exception, et de nombreux exemples montrent que $s^{\prime} y$ développent de nouvelles relations entre ville et agriculture, concourant à penser et fabriquer la ville en intégrant I'agriculture et le jardinage. Là aussi, le pont est fait entre les agriculteurs, la société civile et l'administration, puisque cette dernière a lancé deux groupes de travail sur l'agriculture urbaine1, cherchant à fixer sa place et à définir une politique à son sujet.

Cette insertion de l'agriculture au sens large dans la fabrique et le renouvellement de la ville nous interroge : dans quelle mesure l'agriculture se transforme-t-elle en un outil d'aménagement urbain ? Qui I'utilise ainsi, pour quel(s) type(s) de projet(s) ? Quelles sont les limites de l'agriculture comme outil d'aménagement?

Après une première partie qui vise à définir et identifier ce que l'on peut désigner comme l'agrarisation de la ville à Genève, sera interrogée dans une deuxième partie la capacité de ce processus à devenir instrument d'aménagement urbain. Nous soulèverons en particulier les limites et obstacles législatifs, institutionnels et culturels à l'agrarisation de la ville, qui montrent qu'en dépit d'un changement dans l'imaginaire au sujet du rapport ville-agriculture, et de l'émergence de nombreux projets pilotes d'agriculture urbaine, I'agriculture est encore loin d'avoir transformé la fabrique urbaine.

Cet article se fonde sur une recherche menée dans le cadre du projet "Naville - Les Natures de la Ville ", financé par le Fonds national suisse pour la recherche scientifique (FNS, 2011-2014). Les données de cet article ont été récoltées d'une part à travers une série d'entretiens semi-directifs menés entre septembre 2011 et juin 2013 avec des décideurs locaux, des jardiniers et des agriculteurs, et d'autre part par l'analyse de documents de planification, en libre accès ou fournis par nos partenaires d'entretiens.

\section{VERS UNE AGRARISATION DE LA VILLE}

Urbanisation de l'agriculture et agrarisation de la ville

Deux processus permettent de décrire la relation contemporaine entre agriculture et ville : d'une part I'urbanisation de l'agriculture et d'autre part 
I'agrarisation de la ville (Salomon Cavin, 2012). Le premier processus désigne l'emprise croissante de l'urbanisation sur les espaces et dans les pratiques agricoles. L'expression paraît relativement évidente au vu des discours désormais répandus sur les effets de l'étalement urbain sur les terres agricoles. Le second est plus inhabituel et désigne au contraire l'emprise croissante de surfaces et de pratiques agricoles en ville. II mérite que I'on s'y arrête plus longuement.

\section{L'agriculture urbanisée}

Le premier processus en cours dans la relation ville-agriculture désigne une agriculture qui subit le phénomène d'urbanisation et en est, le plus souvent, la victime.

C'est d'abord l'agriculture des aires urbaines qui devient urbaine malgré elle, du fait de l'emprise croissante de l'urbanisation, et du phénomène d'étalement urbain qui lui est souvent associé (Djellouli et al., 2010); il s'agit d'une agriculture périurbaine peu à peu rattrapée par la ville et soumise au processus d'urbanisation. Dans ce contexte, soit l'espace agricole apparaît comme une réserve foncière pour la ville, soit, rattrapées par l'urbanisation, des surfaces agricoles autrefois bien distinctes des zones habitées se voient peu à peu entourées par les constructions. Les surfaces cultivées forment alors avec les surfaces construites un véritable patchwork. Cette proximité est synonyme d'entrave à l'exploitation : parcelles isolées et éparpillées, concurrence entre voiture et tracteur sur les routes, querelles autour des bruits et des odeurs, excréments canins dans les cultures, etc. La liste est longue des conflits d'usage entre zone à bâtir et zone agricole.

L'urbanisation de l'agriculture a également lieu de manière plus discrète, via les demandes citadines de paysage, d'espaces de loisirs et de détente et de protection de l'environnement (Le Caro, 2007 ; Nahmías et Le Caro, 2012). On entend alors I'urbanisation de l'agriculture non comme un phénomène d'extension de l'objet géographique ville mais comme l'accaparement des espaces agricoles par des pratiques urbaines. II s'agit donc là d'un espace agricole pratiqué par les urbains, participant selon Nahmías et Le Caro (2012) à construire une agricuture urbaine, synonyme de la campagne urbaine de Donadieu (1998). Dans les régions urbanisées les attentes de la population sont particulièrement élevées en ce qui concerne l'accès à des espaces de détente et de loisirs et le maintien de paysages ouverts non encore urbanisés.

Partant, les agriculteurs endossent de manière croissante le rôle de paysagistes et de prestataires de services pour les urbains. Témoin de cette complexification du métier d'agriculteur, le déve- loppement de la notion de multifonctionnalité de I'agriculture (Duvernoy et al., 2005 ; Renting et al, 2009 ; Van Huylenbroeck et Durand, 2003) met en évidence que l'agriculture ne fait pas que produire des produits agricoles mais également des services agricoles. En Suisse par exemple, avec la diminution des subventions à la production et le développement des paiements directs depuis 1998, I'agriculteur est de plus en plus rémunéré pour des services dérivés de l'agriculture comme le paysage et l'environnement ${ }^{2}$.

L'urbanisation de l'agriculture n'est toutefois pas qu'un phénomène négatif pour celle-ci, elle marque aussi l'essor de nouvelles formes d'entreprises agricoles qui saisissent l'opportunité de cette imbrication de plus en plus étroite avec le marché urbain pour court-circuiter les formes traditionnelles de distribution et se rapprocher des consommateurs avides de renouer avec la terre : marchés à la ferme et agriculture contractuelle de proximité se sont remarquablement développés ces dernières années en Suisse Romande. II s'agit là d'une urbanisation fonctionnelle, qui tisse des liens de réciprocité avec la ville, une agriculture tournée vers le marché urbain proche et non vers les marchés de gros. C'est l'agriculture urbaine au sens où Fleury et Donadieu (1997) I'ont définie.

\section{Une agriculture conquérante : I'agrarisation de la ville}

La deuxième facette de la relation actuelle villeagriculture, c'est l'agriculture qui s'insinue en ville. L'agriculture $n^{\prime}$ est donc plus périurbaine mais véritablement intra-urbaine. Ainsi, en même temps que l'agriculture subit la pression urbaine, un processus inverse voit la mise en culture du sol urbain. Cette " agrarisation de la ville " peut prendre des formes très différentes (de la ferme urbaine au jardin communautaire), prendre place sur des supports variés (bâtiments, espaces délaissés, etc.) et recourir à des techniques très diverses (du biologique à I'hydroponique).

Face à la multitude de formes que prend ce phénomène, peut-on et doit-on toujours parler d'agriculture urbaine ? Nous l'avons vu dans la partie précédente, le terme d'agriculture urbaine recoupe des acceptions diverses, qu'il s'agisse de la diffusion dans le monde agricole de pratiques urbaines, ou de la constitution de liens fonctionnels étroits entre I'agriculture et sa ville. En ville, le développement de pratiques culturales est lui aussi protéiforme. Si dans la littérature anglophone le terme d'agriculture urbaine tend à être réservé aux pratiques professionnelles (celui de urban gardening désignant lui le jardinage amateur), en langue française ce terme englobe généralement l'ensemble des pratiques culturales en ville et à ses abords, à condition qu'il y ait une forme de réciprocité entre la ville et son agriculture. L'insertion du jardinage

\section{2 - La Confédération} Helvétique a instauré des paiements directs pour les surfaces de compensation écologique (SCE) lors de sa réforme agricole au début des années 1990. Ces paiements soutiennent les agriculteurs consacrant $7 \%$ de leur exploitation à des SCE telles que prairies extensives, jachères florales, arbres isolés, haies vives ou vergers hautetige. A Genève aujourd'hui, les SCE occupent $12 \%$ de la surface agricole utile cantonale. 
urbain dans cette notion n'est toutefois pas sans poser débat. Grandchamp Florentino (2012) fait état de ces débats et plaide pour une acception large de l'agriculture urbaine, qui n'empêche pas de se pencher sur les spécificités de chaque type de pratiques. Nous I'avons montré ailleurs, en Suisse agriculture et jardinage en ville paraissent être portées par un même imaginaire (Salomon Cavin et Ernwein, à paraître), bien qu'il y ait des spécificités propres à chacun.

Le processus d'agrarisation de la ville permet de rendre compte d'une part du développement en ville de nouveaux types d'agriculture et, d'autre part, de la transformation de la ville par des pratiques agricoles.

Que cela soit par les citadins à travers leurs jardins ou par les architectes, urbanistes et aménagistes qui rêvent de tours agricoles ou de Food Urbanism (Verzone et Dind, 2011), la ville devient un terrain privilégié d'expérimentation de l'agriculture. II s'agit d'une part d'expérimentations techniques, voire technologiques, à l'image de I'hydroponie dans des tours agricoles, ou en tout cas pour le moment dans des bâtiments réaffectés. En Suisse, on en trouve un premier exemple à Bâle avec la réalisation d'une ferme en hydroponie sur un toit (Cossy Wädenswil, 2012). D'autre part, la ville devient le socle d'expérimentations de formes participatives d'agriculture. On songera par exemple à l'expérience des Incredible Edibles de Todmorden (Clarke, 2010), qui expérimente les potagers en libre-service dans la totalité de l'espace urbain comme moyen de proposer un nouveau modèle socio-environnemental pour la ville. Face à un espace agricole en difficulté, la ville apparaît aussi comme le nouveau front agricole, vers lequel les nouveaux agriculteurs non issus de familles d'agriculteurs peuvent tenter de s'installer. En effet, en Suisse notamment, l'espace agricole est pratiquement inaccessible aux personnes sans patrimoine foncier agricole.

L'apiculture urbaine illustre enfin également l'idée d'une agriculture qui se réfugie en ville. En témoignent des titres de journaux évocateurs comme "A Paris, les abeilles ont la belle vie" (RFI, 2009) ou "Les abeilles "réfugiées écologiques" à Paris " (Durand, 2011). En effet, cette pratique est mise en péril à la campagne du fait de la mortalité grandissante des abeilles soumises aux résidus de traitements phytosanitaires. Paradoxalement, la ville semble donc aujourd'hui offrir de meilleures conditions de développement aux abeilles. L'apiculture urbaine dépeint ainsi une ville qui se rend accueillante pour l'agriculture face à une campagne qui serait, elle, délétère.

L'agriculture peut également apparaître comme un outil permettant de changer la ville, tant dans ses dimensions économiques, sociales, environne- mentales que morphologiques.

A la différence du processus d'urbanisation de l'agriculture qui touche principalement les agriculteurs, avec I'agrarisation de la ville l'agriculture devient, en tout cas théoriquement, I'affaire de tous. II s'agit d'un acte souvent militant et multidimensionnel qui vise à résoudre des problèmes désignés comme typiquement urbains. Les discours des porteurs de projets mettent souvent en avant l'agriculture urbaine comme moyen d'arriver à la souveraineté alimentaire des villes dépendantes de circuits d'échanges internationaux (Mougeot, 2006, p. 8), ou des habitants trop dépendants de la grande distribution. Boukharaeva et Marloie (2011) mettent ainsi en évidence I'apport non négligeable du jardinage urbain en Russie à la production agricole nationale, et présentent ces jardins comme des amortisseurs de crise économique. A un niveau plus fin, il s'agit aussi de répondre à des problèmes sociaux associés à la ville moderne et individualiste, en favorisant la création de dynamiques de quartier (Duchemin et al., 2010), en œuvrant pour l'intégration des personnes isolées dans une société urbaine atomisée et dans un marché de l'emploi en crise (Fortier, 2003), ou encore pour le retour à la terre des citadins déconnectés des cycles naturels. Le volet environnemental n'est pas en reste, qu'il s'agisse d'offrir en ville les aménités de la campagne, dans une logique de " plus de campagne en ville, moins de ville à la campagne » (Equiterre, 2011) ou de participer à l'augmentation de la biodiversité urbaine (Irvine, Johnson et Peters, 1999).

Enfin, I'agriculture offrirait de nouveaux potentiels à la ville en permettant de penser de nouvelles morphologies urbaines. Sous le terme générique d'agriurbanisme (Vidal et Fleury, 2009), on retrouve ainsi des projets aussi variés que des agroquartiers (Daiz et al., 2011), ou, à une plus large échelle, les CPULs, Continuous Productive Urban Landscapes (Bohn et Viljoen, 2005). Ceuxci constituent pour l'agriculture ce que les Trames Vertes et Bleues (Arrif et al., 2011 ; Cormier et al., 2010) constituent pour la nature : la pénétration des espaces urbains par des réseaux agricoles de différentes natures et de différentes intensités.

\section{L'agrarisation de la ville dans le contexte genevois}

Depuis les années 2000, la région genevoise fait I'objet d'une attention particulière concernant les relations entre la ville et l'agriculture (Barthassat et al., 2011 ; Niwa, 2009 ; Salomon Cavin, 2011). Cet intérêt est en particulier lié à la place accordée à l'agriculture dans le projet d'agglomération genevois. Ce master plan à l'échelle de l'agglomération est en effet l'un des rares en Suisse, sinon le seul, à intégrer l'agriculture comme composante à part entière du projet urbain. Dans cette partie nous allons détailler la manière dont I'agriculture 
Figure 1 : Genève : commune, canton, agglomération.

Réalisation : Régis Dabrinville, 2014.

a été et est actuellement intégrée dans la ville, et les projets qui $s^{\prime} y$ rapportent. Autrement dit, on $s^{\prime}$ intéresse ici à détailler les formes d'agrarisation de la ville de Genève.

La place et l'importance accordées à l'agriculture à Genève peuvent a priori surprendre mais il est important de noter que la moitié du territoire du canton de Genève est actuellement occupée par des surfaces agricoles (Comité régional francogenevois, 2006). La partie construite du canton accueille des zones urbaines à la densité élevée. Ainsi, selon les statistiques cantonales, la ville de Genève présente une densité de 12000 habitants $/ \mathrm{km} 2$. La préservation d'importantes surfaces agricoles dans le canton a aussi été possible grâce aux possibilités de construire au-delà des frontières cantonales en France (Département de I'Ain et de la Haute-Savoie) et dans le canton de Vaud (région de Nyon). Malgré ce report de l'urbanisation dans les régions voisines, les surfaces agricoles dans le canton de Genève sont actuellement soumises à une forte pression par suite du développement économique et démographique intense de la cité de Calvin. D'ici 2030, la région genevoise prévoit la construction de 100000 logements et de 200000 places de travail. Les pertes pour les surfaces agricoles sont estimées à 2500 ha d'ici 30 ans sur tout le périmètre du projet du Grand Genève (Projet d'agglomération franco-valdo-genevois, 2009, p. 27).

\section{La question agricole, une question centrale pour le canton}

L'espace agricole dans le canton de Genève bénéficie depuis longtemps d'une politique active de protection à la fois dans une visée d'auto-approvisionnement et de protection du paysage.

Du XVIle au XIXe siècle, la Chambre des blés était responsable de la sécurité alimentaire de la population genevoise et devait engranger suffisamment de blé pour nourrir les habitants du canton pendant 2 ou 3 ans. L'écoulement des productions locales en même temps que les prix étaient ainsi toujours assurés par les autorités cantonales (Probst et al., 2004). Outre la promotion de la production alimentaire qui légitimait l'importance agricole du canton, I'agriculture a aussi été soutenue par volonté de préserver le paysage. Dès 1929, des mesures ont été prises pour protéger la ceinture agricole genevoise : située dans la zone 5 du règlement d'affectation des sols, les possibilités de construction y étaient fortement limitées. Seuls quelques riches privilégiés ont ainsi pu construire dans cette zone réservée aux pratiques agricoles. En 1952, cette protection a été renforcée par l'institution d'une véritable zone agricole et ce plus de 20 ans avant que ce zonage soit devenu obligatoire dans le cadre de la loi fédérale sur l'aménagement du territoire (LAT, 1979).
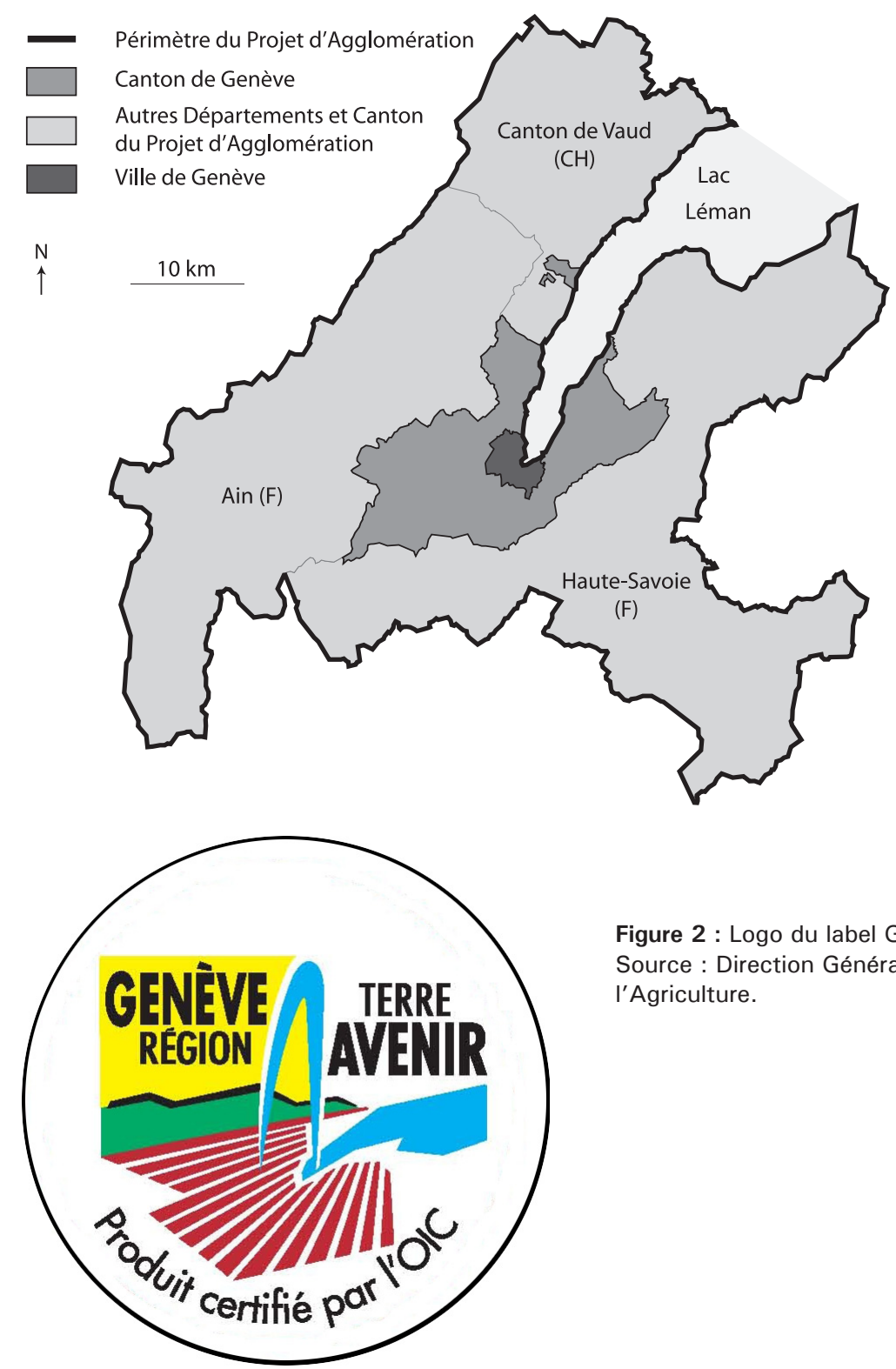

Figure 2 : Logo du label GRTA. Source : Direction Générale de l'Agriculture.
Ainsi, jusqu'à ces dernières années la zone agricole genevoise a pu être largement préservée.

On peut affirmer que cette volonté de préserver le paysage et l'activité agricole du canton est demeurée présente. En témoigne notamment la création du label "Genève Région - Terre Avenir " (GRTA) en 2004.

Ce label, qui promeut toutes sortes de productions locales, est propriété du canton de Genève. Cette mesure tout comme d'autres initiatives $s$ 'inscrit dans le cadre des objectifs de la Loi genevoise sur la promotion de l'agriculture qui vise à « promouvoir une agriculture rémunératrice (...) respectueuse de l'environnement et répondant aux normes sociales et aux besoins du marché et de la population " (Art. 1, al. 1, Loi sur la Promotion de I'Agriculture, LPromAgr, 2004) et à " favoriser les liens entre ville et campagne dans une perspective de plus grande proximité " (Art. 1, al. 2).

Très protégée à l'extérieur de la ville, I'agriculture a également été historiquement présente en ville de Genève, dans les projets comme dans les faits. Dans son plan de développement de Genève de 1929, Maurice Braillard proposait ainsi la création $d^{\prime}$ un maillage $d^{\prime}$ espaces naturels et agricoles comme structure organisatrice d'une future ville immergée dans la nature. La notion $\mathrm{d}^{\prime}$ " espace 
Figure 3 : Localisation des projets mentionnés Réalisation : Régis Dabrinville, 2014.

3 - Parmi les objectifs de ce projet on trouve ainsi la « Promotion des produits de l'agriculture de la région au travers d'actions coordonnées de valorisation des produits locaux " et également " de renforcer certaines filières de production et consolider ainsi la viabilité des exploitations concernée " (Cahier 13-2 Projet agricole d'agglomération - juin 2010, p. 19-20).

4 - http://www.agroquartier. ch/

5 - http://etat.geneve.ch/ $\mathrm{dt} /$ amenagement/actualiteparc agro urbain bernex confignon_exposition concours juin 2013-13822. html

Canton de Genève Communes mentionnées

$\mathrm{N}$<smiles>CSc1ccccc1</smiles>

public agricole " est alors formulée (Barthassat et al., 2011 ; Cogato Lanza, 2003).

Dans le contexte de la pénurie alimentaire liée à la Seconde Guerre Mondiale, Genève de même que toutes les grandes villes de Suisse, est investie par les cultures dans le cadre de la mise en œuvre du Plan Wahlen (Wahlen, 1941). Ce plan élaboré à partir de 1937, visait à la mise en culture de toutes les surfaces disponibles en ville comme à la campagne. En ville, c'est surtout l'utilisation des parcs publics pour la culture qui a marqué les esprits. Ainsi dans les années 1940, les espaces publics de la ville de Genève mis en culture accueillent près de 4000 parcelles de jardins potagers. A I'issue de la guerre, près de la moitié disparaît les autres prennent la dénomination de jardins familiaux. Aujourd'hui sur la totalité du canton on compte encore quelque 2200 jardins familiaux, toutefois désormais principalement situés dans les franges urbaines. En effet, à quelques exceptions près, ils suivent le front d'urbanisation, marquant une transition vers l'espace agricole plus qu'une agrarisation de la ville.

Diverses pratiques agricoles urbaines ont donc occupé à plusieurs reprises une place importante dans I'histoire et l'identité locale, avec des déclinaisons multiples. Cette dynamique se poursuit sous d'autres formes actuellement.

\section{Planification et agrarisation}

On peut actuellement distinguer deux échelles de projet où se pense l'intégration de l'agriculture dans la fabrique urbaine à Genève : le grand territoire (région urbaine) et le quartier.

Rare exemple en Suisse, l'agriculture à Genève est donc intégrée dans la planification directrice à l'échelle de l'agglomération du Grand Genève. La particularité du projet est également son caractère transfrontalier. Par conséquent, ce sont deux systèmes agricoles relativement distincts qui sont pris en compte dans ce vaste territoire de $2000 \mathrm{~km}^{2}$. L'intégration de l'agriculture dans le projet a commencé par une suite de diagnostics sur la situation de l'agriculture de part et d'autre de la frontière (Comité régional franco-genevois, 2006 ; Projet d'agglomération franco-valdo-gene- vois, 2009 ; Projet d'agglomération franco-valdogenevois, 2011). Dans le même temps, et à leur demande, les milieux agricoles (représentants des filières, de la chambre d'agriculture etc.) ont été intégrés au processus participatif de construction du projet. Concrètement, I'agriculture a vu sa place dans le projet évoluer. Dans le cadre du premier projet d'agglomération (2003-2007), c'est via le Plan Paysage, c'est-à-dire de manière relativement traditionnelle que l'agriculture a été identifiée comme "socle du projet urbain " (Barthassat et al., 2011). Dans un deuxième temps, « le Projet Agricole d'Agglomération " (Cantiniaux et Dériaz, 2010) propose pour l'agriculture un projet urbain qui lui est expressément dédié. La place désormais accordée à l'économie agricole marque un tournant dans la manière $d$ 'aborder l'agriculture dans le projet urbain non plus seulement comme paysage mais également comme activité économique $^{3}$.

Ainsi, un changement notoire est à relever dans la manière de considérer du point de vue de l'aménagement urbain l'espace agricole qui ceinture la ville. Une part importante du projet d'agglomération est en effet consacrée à l'analyse de l'espace agricole et aux conditions agricoles nécessaires pour en assurer la pérennité. En conséquence, audelà même du paysage reconsidéré comme socle du projet, le master plan intègre également des objectifs qui concernent la production agricole.

La question agricole est désormais également présente dans les réflexions qui accompagnent la création de nouveaux quartiers à Genève. Une des premières tentatives de lier agriculture et urbanisme a concerné le projet de quartier des Cherpines (fig. 3), situé dans une plaine agricole dans le prolongement immédiat de zones densément urbanisées de Genève. L'urbanisation de cet espace, actuellement encore agricole, a donné lieu à un débat houleux, démarré en 2008 avec l'étude de faisabilité, et qui s'est soldé par un référendum en mai 2011. La population appelée à s'exprimer sur le bien-fondé de ce projet a accepté à une courte majorité que cette zone soit urbanisée (Matthey et Weil, 2013 ; Salomon Cavin, 2012). Ce projet va entrainer la disparition ou le déplacement de plusieurs entreprises agricoles. Dans les divers scénarios de développement pour cette 
zone, l'une des esquisses proposait de marier pratiques agricoles et développement urbain au sein d'un agroquartier. Parallèlement au concours et au lancement du référendum, I'association Agro Quartiers Autogérés est également créée ${ }^{4}$. En dépit de ces diverses propositions, l'idée générale d'un agroquartier n'a pas été retenue pour le projet. En revanche, il est désormais prévu des espaces dédiés aux cultures à hauteur de $6 \mathrm{~m} 2$ par habitant. La réflexion porte actuellement sur la mise en œuvre de cet objectif. Deux projets semblent se dessiner : soit un ensemble de jardins disséminés dans toute la zone, soit une zone agricole centrale d'environ 1,5 ha. Dans ce dernier cas, il y aurait la possibilité de la confier à un exploitant agricole. Le concept même d'agroquartier est assez flou mais il est intéressant de constater que la question des cultures et du jardinage est désormais extrêmement présente dans les nouveaux projets de quartier à Genève.

Constatant que "I'agriculture est une composante qui s'invite clairement dans la composition urbaine des nouveaux quartiers", la Direction générale de l'agriculture (organe cantonal) a ainsi lancé une réflexion sur la possibilité de développer l'usage agricole dans des espaces sis dans les nouveaux quartiers urbains : "Ces espaces de production devront être trouvés dans le cadre de la composition urbaine, en remplacement d'un espace vert traditionnel (pelouse) et non en supplément sur la zone agricole ", " un producteur pourrait conduire une exploitation agricole en collaboration avec des "jardiniers-consommateurs-habitants" du quartier projeté "(Ansaldi, 2011). Un tel projet est actuellement à l'étude dans le cadre d'un futur quartier de la commune de Bernex (fig. 3). Suite à un concours d'idée, un " parc agro urbain $\|^{5}$ (de 2-3ha) sur I'un des trois espaces publics ouverts prévus a d'ores et déjà été choisi.

\section{Agrarisation et requalification urbaine}

En dehors des projets urbains proprement dits, qui consistent à créer du bâti où il $n^{\prime} y$ en avait pas, Genève comme les autres villes européennes se re-construit aussi sur elle-même. L'agriculture dans sa modalité jardinière tend à être mobilisée dans ces projets, où interviennent différents acteurs institutionnels. La question de l'usage des jardins collectifs dans leur multiplicité de formes et d'acceptions (relief gardens, war gardens et victory gardens notamment) comme outil d'urbanisme au fil du $X X^{e}$ siècle aux Etats-Unis a été analysée par Lawson (2004), qui met en évidence une ambiguïté : ces jardins sont successivement mobilisés tout au long du $X X^{e}$ siècle pour répondre à de multiples problèmes sociaux, mais ne sont jamais pérennisés, apparaissant toujours comme des outils d'intervention ponctuels et éphémères. A Genève, aujourd'hui, se développe une nouvelle politique du jardinage qui cherche justement à dépasser le modèle historique du jardin familial et à penser une politique plus intégrée et pérenne du jardinage urbain, à travers les potagers urbains, communément dénommés " plantages".

Selon les cas, les acteurs qui mobilisent les jardins urbains comme outil de requalification urbaine sont issus des services sociaux, des services des espaces verts, ou encore du service Agenda 21. En ville de Genève, ces acteurs ont constitué un groupe de travail sur la question, dans le but d'identifier les espaces propices à ce genre de projet et de proposer une politique commune. Les espaces cibles sont principalement les pieds d'immeuble. Les arguments de ces porteurs de projet institutionnels sont multiples. Ces espaces seraient souvent sous-utilisés et feraient l'objet de dégradations. Leur rénovation et leur réattribution à du jardinage permettrait en sus de recréer du lien social où l'on suspecte qu'il n'y en ait plus, de redonner une meilleure image à des quartiers mal vus, et de sécuriser les lieux (dans la commune suburbaine de Thônex par exemple, le tout récent potager urbain (fig. 3) remplace une forêt de bambous souvent désignée comme un coupegorge). Un des responsables du service social prenant part au groupe de travail insiste sur la notion de conquête de l'espace par ces pratiques jardinières (entretien, service social, novembre 2012). On le voit, les pratiques jardinières et agricoles conquièrent les espaces et les projets.

On trouve aussi à Genève quelques exemples de requalification urbaine "spontanée " qui mobilisent I'agriculture. L'exemple archétypal est le Collectif Beaulieu (fig. 3). Ce Collectif s'est réapproprié, en 2010, I'ancien centre de production horticole du Service des espaces verts de la Ville de Genève, situé dans le Parc Beaulieu, en centreville. Les porteurs de projet ont, au départ, souhaité se réapproprier un espace délaissé afin de l'utiliser comme lieu d'expérimentation agricole et d'interpellation des citadins sur la situation de I'agriculture et la question de l'industrialisation des semences. Aujourd'hui, leur présence est reconnue et subventionnée par la municipalité. Mais le parc va prochainement être réaménagé. Une interrogation est donc en cours sur la place de ce type de projets dans un parc en centre-ville. Un des arguments pour le maintenir est la possibilité $d^{\prime}$ en faire une vitrine de l'agriculture locale.

\section{L'AGRARISATION DE LA VILLE : (NOUVEL) OUTIL D'AMÉNAGEMENT URBAIN ?}

Ces différentes expériences - plus ou moins pérennes, plus ou moins abouties - qui correspondent à ce que nous nommons agrarisation de la ville démontrent la montée en puissance d'une ville dont le modèle, la fabrique et l'usage renouent avec l'agriculture. Désormais, dans le contexte genevois, pas un nouveau quartier n'est dessiné sans 
7 - A Genève, la ferme de Budé (fig. 3) est ainsi située dans une parcelle du domaine public de la zone à bâtir. que I'on y prévoie un espace en culture ou une relation d'achalandage privilégiée avec l'espace agricole avoisinant. Aussi peut-on légitimement se demander dans quelle mesure l'agriculture est en passe de devenir un nouvel outil d'aménagement de la ville.

\section{Ville compacte et agrarisation de la ville}

L'objectif de limiter l'étalement urbain est-il compatible avec le processus d'agrarisation de la ville?

L'idée de l'agriculture comme outil d'aménagement urbain n'est pas vraiment nouvelle en Suisse si I'on s'en tient au principe d'un espace agricole comme ultime rempart contre I'urbanisation. La loi fédérale sur l'aménagement du territoire (LAT, 1979) stipule en effet la séparation entre zone à bâtir et zone de non bâtir. Cette dernière est désignée comme zone agricole (art. 16). Le développement des constructions y est fortement réglementé. Ce zonage agricole a constitué un outil très efficace pour la réalisation de I'objectif premier de la LAT : I'utilisation mesurée du sol, largement synonyme en Suisse de limitation de l'étalement urbain. Comme souligné ci-dessus, le canton de Genève a très tôt mobilisé l'agriculture pour limiter la croissance urbaine. Cette fonction-là de I'agriculture demeure à I'heure actuelle toujours dominante dans sa relation avec l'aménagement urbain : I'espace agricole définit le front urbain.

Dans le cas du projet de Bernex, mentionné plus haut, la négociation se rapportant à l'agriculture concernait avant tout la limitation de l'emprise du projet sur l'espace agricole. C'est dans cette optique que la surface des parcs publics initialement prévus a été diminuée. C'est seulement dans un second temps, au moment où l'emprise de l'urbanisation sur les surfaces agricoles était connue et acceptée, que l'idée d'un usage agricole au sein des espaces publics prévus a été développée (entretien, DGA, 2013).

Comme I'ont souligné nombre de nos interlocuteurs, I'intégration de l'agriculture dans I'aménagement des espaces (intra-) urbains ne doit pas se faire au prix de l'étalement urbain.

\section{Un cadre règlementaire inadapté}

La règlementation relative à l'agriculture est pour le moment un frein au développement de l'agriculture dans les zones urbanisées. Actuellement I'espace règlementairement dédié à l'activité agricole est la zone agricole (art. 16, LAT, 1979). Ce zonage limite drastiquement les types de bâtiments qui peuvent y être construits. Seules les constructions en relation directe avec la pratique agricole sont ainsi tolérées. Réciproquement, I'usage agricole d'espaces sis en zone à bâtir n'est pas du tout prévu par la loi. Seul le jardinage, au sens de production agricole non marchande est possible. Des exceptions existent mais elles sont très rares6.

L'usage agricole de certaines zones urbaines apparaitrait pourtant pour certains agriculteurs comme une alternative possible pour développer de petites unités agricoles dont la forme d'exploitation (type panier contractuel) permet une viabilité de petites unités (2 ha). Mais, pour toute personne souhaitant devenir exploitant, l'accès à la terre est difficile voire impossible si celle-ci n'est pas issue d'une famille d'agriculteurs ou propriétaire de parcelles en zone agricole. En effet, le droit foncier rural (LDFR, 1991), et dans un but estimable de protection des surfaces en exploitation, limite drastiquement les possibilités d'accès à la terre. Une agriculture qui reste à définir

Enfin, un obstacle majeur à la transformation de I'agriculture en un outil d'aménagement urbain réside dans le flou conceptuel et les conflits terminologiques autour de l'agriculture urbaine et dans les représentations que se font les multiples acteurs de l'agriculture et de la ville.

L'agriculture en ville n'est pas l'agriculture conventionnelle, celle du sens commun. II s'agit de nouvelles formes d'agriculture, souvent participatives, amatrices ou semi professionnelles, qui ne sont pas organisées autour de la figure unique de l'agriculteur. Le Collectif Beaulieu, par exemple, déjà présenté précédemment, développe un projet multidimensionnel, constitué de la production de plantons pour les ACP (Agriculture contractuelle de proximité, équivalent des AMAP françaises) du canton, de la vente de plantons au public, d'un jardin de cueillette, et de l'accueil de projets divers (apiculture, micro élevage de poules, fabrication de tisanes, projet de guerrilla gardening, fabrique de conserves et de bière, banque de graines, ainsi que, depuis 2013, potagers urbains pour les habitants du quartier...). On le voit, se retrouvent mêlés dans ce projet des productions différentes, des acteurs variés et des logiques multiples, allant de I'approvisionnement des ACP à I'autoproduction familiale en passant par un volet éducatif et expérimental.

Par rapport aux formes traditionnelles de I'agriculture qui se situent en zone agricole, I'agrarisation de la ville participe à la construction de pratiques culturales qui concernent d'autres acteurs, d'autres logiques et d'autres objectifs, ou qui les mixent. On parle donc d'une agriculture aux contours difficilement définissables, ce qui peut poser problème pour le développement de politiques.

Loin d'être anodines, les questions terminologiques et de définition sont porteuses d'enjeux. A Genève, la Direction générale de I'Agriculture 
a rédigé en 2012 un "Lexique de l'agriculture urbaine " (Direction générale de l'Agriculture 2012) à destination des acteurs agricoles et urbains, dans lequel figuraient sous I'appellation agriculture urbaine aussi bien l'agriculture périurbaine, l'agriculture intraurbaine, le jardinage urbain, que le jardinage dans les jardins privatifs résidentiels. Soumis à la consultation auprès de milieux agricoles et urbanistiques, le lexique a rapidement été transformé en "Lexique genevois. La production agricole d'agglomération " (Direction générale de I'Agriculture 2012). II a en effet fait face à un vent de critiques : les agriculteurs tiennent à différencier leurs pratiques professionnelles des pratiques amatrices de jardinage et ne souhaitent pas voir qualifier leur pratique d'urbaine ; quant aux urbanistes de la ville, pour la plupart d'entre eux I'agriculture au sens propre ne peut se faire en zone urbaine mais en zone agricole. On le voit, la notion d'agriculture pose ici autant question que celle de ville.

\section{CONCLUSION}

II existe aujourd'hui de nombreux projets qui permettent de définir les contours de l'agrarisation de la ville à Genève. Cette agrarisation, ou conquête du sol et du projet urbains par des pratiques agricoles, y prend des formes multiples, de la ferme urbaine au potager urbain, en passant par les projets d'agroquartier. Elle se retrouve à différents niveaux du projet urbain, de la planification territoriale à l'échelle de la région urbaine, comme à celui de la requalification du tissu urbain. II s'agit d'un processus porté par de multiples acteurs, issus tant de la société civile et du monde associatif que de l'administration. Cette multitude d'acteurs ainsi que les groupes de travail constitués dans les administrations illustrent l'intérêt porté par les acteurs de la ville à cette question, et sa potentielle portée comme politique urbaine transversale. On peut donc penser que l'agriculture devient ou deviendra outil de réaménagement des villes.

Cependant, I'agrarisation de la ville pose plusieurs problèmes. L'idée de l'agriculture comme outil $\mathrm{d}^{\prime}$ aménagement urbain se heurte à la conception traditionnelle du zonage qui place exclusivement I'agriculture en zone agricole. Dans I'aménagement urbain, le rôle principal de l'agriculture demeure celui de ceinture verte de l'urbanisation. La distinction franche entre zone urbaine et zone agricole demeure le repère incontournable de l'aménagement du territoire. En outre, I'agrarisation de la ville pose problème quand elle mêle agriculture et jardinage. Les acteurs agricoles ne veulent pas être associés au jardinage amateur car ils souhaitent défendre leur profession qu'ils considèrent déjà en danger. Par conséquent ils se montrent suspicieux vis-à-vis de projets dits $d^{\prime}$ agriculture urbaine qui justifieraient, sous couvert de développer des potagers urbains, de dé- classer des zones agricoles en zone à bâtir. Leur crainte est que l'agriculture urbaine fasse écran à leurs problèmes.

Enfin, le volet agricole à proprement parler est finalement aussi rejeté avec plus ou moins de vigueur par certains acteurs parce qu'il ne répondrait pas à des problèmes urbains, ou en tout cas pas à ceux de la majorité des habitants de la ville. Dans le cadre du projet de réaménagement du parc Beaulieu, différents acteurs ont souligné que I'agriculture est une thématique trop spécialisée pour un espace public, car elle appelle seulement un public intéressé et souvent politisé ; c'est une thématique trop éloignée des problématiques des habitants des quartiers, ce qui la rend élitiste et donc excluante (entretien, ville de Genève, 2012). Or un espace public ne devrait pas être réservé à une communauté d'intérêts. En revanche le jardinage urbain, sous la forme de potagers urbains collectifs ou "plantages ", est vu comme un bon outil pour réunir les habitants autour d'un projet collectif, car il demande peu de connaissances et d'investissement. Le jardinage serait un instrument adapté pour répondre aux problèmes (des habitants) urbains du fait de sa proximité avec la notion de nature en ville (Niwa, 2009). L'agriculture, elle, répondrait à des problématiques plus vastes non spécifiques à la ville.

Ce dernier exemple démontre finalement que si le jardinage urbain se conjugue bien à la notion de nature en ville et s'inscrit dans une histoire sociale connue, l'agriculture demeure associée, dans les représentations comme dans les pratiques, à une activité hors la ville.

\section{BIBLIOGRAPHIE}

ANSALDI E., 2011, Une agriculture intra-urbaine professionnelle à Genève. Projet de cahier des charges, Direction générale de I'agriculture, Novembre 2011 (non publié).

ARRIF T., BLANC N., CLERGEAU P., 2011, Trame verte urbaine, un rapport Nature-Urbain entre géographie et écologie, Cybergeo: European Journal of Geography, article n ${ }^{\circ} 574$.

BARTHASSAT M., BEUCHAT S., DERIAZ G., 2011, Agriculture, ville et paysage : enjeux du projet d'agglomération franco-valdo genevois, Urbia, $\mathrm{n}^{\circ} 12$, p. 107-136.

BOUKHARAEVA L., MARLOIE M., 2011, L'utilisation des sols par le jardinage urbain à grande échelle de Russie, Économie rurale, vol. 5, n³25-326, p. 176-191.

CANTINIAUX A.-L., DÉRIAZ G., 2010, Projet agricole d'agglomération, Cahier 13.2. 
LE CARO Y., 2007, Les loisirs en espace agricole. L'expérience d'un espace partagé, Rennes, Presses universitaires de Rennes, $431 \mathrm{p}$.

CLARKE P., 2010, Incredible Edible: how to grow sustainable communities, FORUM, vol. 52, p. 69-76.

COGATO LANZA E., 2003, Maurice Braillard et ses urbanistes. Puissance visionnaire et stratégies de gestion urbaine (Genève 1929-1936), Genève Slatkine, $297 \mathrm{p}$.

COMITÉ RÉGIONAL FRANCO-GENEVOIS, 2006, Diagnostic de l'espace agricole transfrontalier franco-valdo-genevois.

CORMIER L., LAJARTRE A. B. D., CARCAUD N., 2010, La planification des trames vertes, du global au local: réalités et limites, Cybergeo: European Journal of Geography, Document 504.

COSSY WÄDENSWIL C., 2012, L'agriculture du futur passe par les villes, Le Temps, avril.

DAIZ A., FRANÇOIS P., VILLALBA L., 2011, Dessine-moi un agroquartier, Mémoire réalisé dans le cadre du cours PTI : agriculture urbaine, Master en Géosciences de l'environnement, Université de Lausanne (non publié), $39 \mathrm{p}$.

DIRECTION GÉNÉRALE DE L'AGRICULTURE, 2012, Lexique genevois sur l'Agriculture Urbaine, (non publié).

DIRECTION GÉNÉRALE DE L'AGRICULTURE, 2012, Lexique genevois. La production agricole d'agglomération, (non publié).

DJELLOULI Y., EMELIANOFF C., BENNASR A., CHEVALIER J., 2010, L'étalement urbain. Un processus incontrôlable?, Rennes, Presses universitaires de Rennes, $260 \mathrm{p}$.

DONADIEU P., 1998, Campagnes urbaines, Arles, Actes Sud et ENSP, $219 p$.

DUCHEMIN E., WEGMULLER F., LEGAULT A.M., 2010, Agriculture urbaine : un outil multidimensionnel pour le développement des quartiers, [VertigO] La revue électronique en sciences de l'environnement, vol. $10, \mathrm{n}^{\circ} 2$.

DURAND A.-A., 2011, Les abeilles "réfugiées écologiques" à Paris, Metro, 14 Septembre.

DUVERNOY I., JARRIGE F., MOUSTIER P., SERRANO J., 2005, Une agriculture multifonctionnelle dans le projet urbain : quelle reconnaissance, quelle gouvernance?, Les Cahiers de la multifonctionnalité, vol. 8, p. 87-104.
EQUITERRE, 2011, Des potagers urbains : pour jardiner au pied de chez soi et colorer nos villes !, Equiterre Info, février, vol. $11, \mathrm{n}^{0} 1$.

FLEURY A., DONADIEU P., 1997, De I'agriculture périurbaine à l'agriculture urbaine, Courrier de I'environnement de I'INRA, vol. 31, p. 45-61.

FORTIER A., 2003, Les vertus du jardinage d'insertion, Communications, vol. 74, $\mathrm{n}^{\circ} 1$, p. 85-102.

GRANDCHAMP FLORENTINO L., 2012 L'agriculture urbaine : Un enjeu de la ville durable, Revue des sciences sociales, $\mathrm{n}^{\circ} 47$, p. 142-152.

VAN HUYLENBROECK G., DURAND G., 2003 Multifunctional Agriculture: A New Paradigm for European Agriculture and Rural Development, Hampshire, Ashgate, $239 \mathrm{p}$.

IRVINE S., JOHNSON L., PETERS K., 1999, Community gardens and sustainable land use planning: A case study of the Alex Wilson community garden, Local Environment, vol. 4, $\mathrm{n}^{\circ} 1$, p. 33-46.

LAT, 1979, Loi sur l'Aménagement du Territoire, juin.

LAWSON L., 2004, The planner in the garden: A historical view into the relationship between planning and community gardens, Journal of Planning History, vol. $3, \mathrm{n}^{\circ} 2$, p. 151-176.

LPROMAGR, 2004, Loi sur la Promotion de I'Agriculture.

MATTHEY L., WEIL M., 2013, Urbain-rural : reconfiguration du jeu d'acteurs, Collage - revue de la fédération suisse des urbanistes, vol. $1, \mathrm{n}^{0} 13$, p. 19-22.

MOUGEOT L. J., 2006, Cultiver de meilleures villes : agriculture urbaine et développement durable, Ottawa, Centre de recherches pour le développement international, $113 \mathrm{p}$.

NAHMÍAS P., LE CARO Y., 2012, Pour une définition de l'agriculture urbaine: réciprocité fonctionnelle et diversité des formes spatiales, Environnement urbain/Urban Environment, vol. 6, p. 1-16.

NIWA N., 2009, La nature en ville peut-elle être agricole? De la Suisse au Japon, Urbia, $\mathrm{n}^{\circ} 8$, p. 103-126.

PROBST V., SANTSCHI C., OBERWILER E., BERGUER F., ZUMKELLER D., CSILLAGI J., 2004, Entre la chèvre et le chou: (a)ménagements agricoles, Genève, Archives d'Etat, $44 \mathrm{p}$. 
PROJET D'AGGLOMÉRATION FRANCO-VALDOGENEVOIS, 2009, Analyse des impacts du projet d'agglomération sur l'agriculture, Rapport d'étape, Cahier 13.1.

PROJET D'AGGLOMÉRATION FRANCO-VALDOGENEVOIS, 2011, Cartographie des espaces agricoles fonctionnels, protégés réglementairement et des zones de frottement avec le projet d'agglo. Cahier 13.23 .

RENTING H., ROSSING W., GROOT J., VAN DER PLOEG J., LAURENT C., PERRAUD D., STOBBELAAR D. J., VAN ITTERSUM M., 2009, Exploring multifunctional agriculture. A review of conceptual approaches and prospects for an integrative transitional framework, Journal of Environmental Management, vol. 90, $\mathrm{n}^{\circ} 2$, p. $112-123$.

RÉPUBLIQUE ET CANTON DE GENÈVE, 2001, Plan Directeur Cantonal.

RFI, 2009, A Paris, les abeilles ont la belle vie, www.rfi.fr, août.

SALOMON CAVIN J., 2012, Entre ville stérile et ville fertile, I'émergence de l'agriculture urbaine en
Suisse, Environnement urbain/Urban Environment, vol. 6, p. 17-31.

SALOMON CAVIN, J., ERNWEIN, M., à paraître, La ville fertile : nouveau mythe fondateur pour l'agriculture urbaine à Genève ?, Actes du colloque Nature Urbaine en Projets.

SALOMON CAVIN J., RUEGG J., à paraître, Retisser les liens entre agriculture et ville, in VAYSSIÈRE B et GAILLARD D. (eds), Orienter Genève vers la durabilité : enjeux et pistes de réflexion, Genève, Fondation Braillard Architectes In Folio.

VERZONE C., DIND J.-P., 2011, De I'agriculture urbaine au food urbanism : état des lieux et perspectives pour la Suisse, Urbia, $n^{\circ} 12$, p. 137-160.

VIDAL R., FLEURY A., 2009, Aménager les relations entre la ville et I'agri-culture. De nouveaux enjeux territoriaux et une nouvelle approche "agriurbaniste," Urbia, n8, p. 127-142.

WAHLEN F.-T., 1941, La bataille des champs. L'agriculture suisse en temps de guerre, Neuchâtel, La Baconnière, $31 \mathrm{p}$. 